\title{
Caveolin-1 expression in thyroid neoplasia spectrum: Comparison of two commercial antibodies
}

\author{
Jelena Jankovića , Svetlana Paskaša $^{\mathrm{a}}$, Ilona Marečko ${ }^{\mathrm{a}}$, Vesna Božićb ${ }^{\mathrm{b}}$, Dubravka Cvejića and \\ Svetlana Savin ${ }^{\text {a,* }}$ \\ ${ }^{a}$ Institute for the Application of Nuclear Energy - INEP, Department for Endocrinology and Immunoradiology, \\ University of Belgrade, Belgrade, Serbia \\ ${ }^{\mathrm{b}}$ Clinical Center of Serbia, Department of Endocrine and Cardiovascular Pathology, Belgrade, Serbia
}

\begin{abstract}
We evaluated caveolin-1 expression in the human thyroid neoplasia spectrum with the aim of examining differences in expression as detected by two anti-caveolin-1 antibodies, and secondly, to investigate the association of caveolin-1 expression levels with aggressive papillary thyroid carcinoma (PTC). Immunohistochemical staining using sc894 or AV09019 antibodies revealed that caveolin-1 was generally overexpressed in the PTC group as a whole (classical and follicular variant) when compared to peritumoral tissue (PT), while it was not detected in about half of follicular thyroid carcinoma (FTC) and majority of follicular adenomas (FTA). Caveolin-1 expression decreased in the following order: clPTC, fvPTC, FTC, PT and FTA. The diagnostic accuracy of AV09019 was better than that of sc894 for discriminating: FTA from FTC, FTA or FTC from the follicular variant of PTC, total PTC from nonmalignant tissue, and malignant tumors from nonmalignant tissue. Spearman's analysis revealed positive correlations of caveolin-1 expression and extrathyroidal invasion $(p<0.05)$ in PTC for both antibodies. Additionally, AV09019 antibody correlated caveolin-1 upregulation with pathological T status.

To conclude, as an immunohistochemical marker AV09019 antibody performed better than sc894 in distinguishing certain histotypes of thyroid tumors. In addition, increased expression of caveolin-1 may be considered as an indicator of papillary carcinoma progression.
\end{abstract}

Keywords: Thyroid tumors, caveolin-1, sc894 (Santa Cruz Biotechnology) antibody, AV09019 (Sigma-Aldrich) antibody, immunohistochemistry

\section{Introduction}

Tumors of the thyroid gland, arising from thyroid follicular epithelial cells, are one of the most common endocrine neoplasms, and their incidence is on the rise worldwide [1-4]. Follicular patterned thyroid tumors share cytological and histomorphological features, so distinguishing a malignant tumor from its benign counterpart is a particular problem in thyroid oncology. Un-

\footnotetext{
* Corresponding author: Svetlana Savin, Institute for the Application of Nuclear Energy - INEP, Department for Endocrinology and Immunoradiology, University of Belgrade, Banatska 31b, P.O.Box 46, 11080 Zemun - Belgrade, Serbia. Tel.: +381 112618 666; Fax: +381 112618 724; E-mail: ssavin@inep.co.rs.
}

like the classical variant of papillary thyroid carcinoma (clPTC), the morphology of which is easily categorized, the follicular variant of papillary carcinoma (fvPTC), follicular carcinoma (FTC) as well as follicular adenoma (FTA) are often difficult to tell apart. Thus, the identification of reliable markers of thyroid malignancy that would facilitate differential diagnostics or improve prognostic predictability of thyroid tumors is of great importance.

Recent studies have shown that caveolin-1, a $22 \mathrm{kDa}$ integral membrane protein could be useful for differentiation of certain types of thyroid tumors $[5,6]$. Caveolin-1 belongs to a family of proteins (cav-1, cav2 and cav-3) that are key components of flask-shaped membrane invaginations called caveolae. These struc- 
tures are formed through oligomerization of caveolins (cav-1 or cav-3) and their association with cholesterolrich lipid-raft domains [7]. Caveolin-1 is expressed in a wide range of tissues, particularly in endothelial cells, adipocytes, fibroblasts and smooth-muscle cells [8]. Caveolin functions as a scaffolding protein in caveolae and is implicated in a variety of processes including: vesicular transport, cholesterol homeostasis [9], and the regulation of intracellular signaling pathways via interactions with raft-localized signaling proteins [10]. Depending on the interaction molecule, cav-1 modulates different cellular signaling pathways and is indirectly implicated in the regulation of both cell proliferation and cell death $[11,12]$.

Altered levels of expression of caveolin-1 in a variety of human malignancies, reflect its dual role in carcinogenesis, as well. It has been found that expression varies between tumor type and stage, resulting in caveolin-1 being defined as both a tumor suppressor and a tumor promoter. As reviewed by Williams and Lisanti [13] down-regulation of caveolin-1 was observed in breast, lung, ovarian cancer, many sarcomas, some adenocarcinomas, whereas in squamous cell carcinomas and multidrug resistant human cancer cell lines, caveolin-1 levels were upregulated. Expression of caveolin- 1 correlates positively with a favorable prognosis of bile duct and salivary gland tumors [14, 15], but is also associated with poor prognosis, disease recurrence, drug resistance and invasion or metastasis in prostate [16], breast [17], lung and brain cancer [18, 19].

Caveolin-1 is expressed in normal thyroid tissue [20] but overexpression in papillary thyroid carcinomas and underexpression in follicular thyroid carcinoma and adenoma have been reported [6,21], suggesting its potential use as a marker in thyroid diagnostic pathology.

The aim of this study was to evaluate the competence of two caveolin-1 antibodies in differential diagnostics of thyroid tumors. In order to explore the potential of caveolin-1 as a biomarker we (i) conducted an immunohistochemical study of caveolin-1 expression in thyroid lesions, (ii) evaluated the ability of each marker to differentiate certain thyroid tumor histotypes, and (iii) correlated these findings with several clinicopathological features of papillary thyroid cancer patients.

\section{Material and methods}

\subsection{Patients and tissue samples}

All clinical specimens used in this study were obtained with Institutional Review Board approval and informed consent to use excess biological material for investigative purposes from all patients participating in the study. On the basis of the availability of tissue samples in the archives of the Clinic for Endocrinology, Diabetes and Diseases of Metabolism, Clinical Center of Serbia, Belgrade, 116 patients with thyroid neoplasia between 2001 and 2010 were identified and analyzed. Cases comprised 30 follicular thyroid adenomas (FTA), 67 papillary thyroid carcinomas (PTC), among which 33 were classical type (clPTC) and 34 follicular type (fvPTC), and 19 follicular thyroid carcinomas (FTC) giving a total number of 116 cases. Peritumoral tissue (PT) originating from non-neoplastic tissue was taken from the following tumor types: 20 from the 30 FTAs, 10 from the 19 FTCs and 17 from 33 clPTC and 15 from the 34 fvPTC. The original whole-section slides and/or re-cuts were verified by two pathologists to establish gold standard diagnoses. All available clinical data, surgical and pathology reports were recorded. Carcinomas were staged in accordance with the American Joint Committee on Cancer (AJCC) Tumor Node Metastasis (TNM) system.

\subsection{Immunohistochemistry (IHC)}

Immunohistochemistry was performed as described previously [22]. The following primary antibodies were used for IHC staining: polyclonal rabbit anticaveolin-1 sc894 (Santa Cruz Biotechnology Inc., Santa Cruz, CA, USA) and polyclonal rabbit anti-caveolin1 AV09019 (Sigma-Aldrich, St. Louis, MO, USA), both diluted 1:300. The signal was enhanced by avidinbiotin-peroxidase complex (Vectastain ABC kit, Vector Laboratories, Burlingame, CA, USA), followed by visualization of the reaction with 3,3'-diaminobenzidine tetrahydrochloride (DAB) solution (Peroxidase Substrate Kit, Vector Laboratories, Burlingame, CA, USA). Controls were incubated with PBS in place of the primary antibody and no positive staining was observed. We reported the results in a semiquantitative fashion, that is, no or focal staining $(0)$, weak $(+1)$, moderate $(+2)$, and strong $(+3)$ diffuse cytoplasmic staining of thyrocytes. Staining of endothelial cells and lymphocytes served as internal positive and negative controls, respectively.

\subsection{Western immunoblotting $(W B)$}

For WB analysis, fresh tissue samples taken from both cancer and matched non-cancerous tissue were immediately snap-frozen in liquid nitrogen, and stored at 
$-80^{\circ} \mathrm{C}$ until protein was extracted. Aliquots $(100 \mathrm{mg})$ of frozen tissue samples were minced with scissors, then cut into smaller pieces at $4{ }^{\circ} \mathrm{C}$ with addition of Protease Inhibitors Cocktail (P8340, Sigma-Aldrich, St. Louis, MO, USA) and homogenized in lysis buffer (20 mM TRIS-HCl pH 8, 137 mM NaCl, 10\% glycerol, $1 \%$ Nonidet-P 40, 2 mM EDTA), and centrifuged for $10 \mathrm{~min}$ at $4^{\circ} \mathrm{C}$ at $11000 \mathrm{rcf}$. The protein content was determined conventionally using a BCA Protein Assay Kit (Pierce Biotechnology, Rockford, IL, USA).

Proteins (50 $\mu \mathrm{g}$ per lane) were separated by SDSPAGE on $15 \%$ PAA gel in reducing conditions. Proteins were transferred to polyvinylidene fluoride membrane (Millipore, Billerica, MA, USA) and blocked with 5\% casein in PBS. Prior to blocking, membranes were stained with Ponceau $\mathrm{S}$ to validate equal loading of the lanes. Immunoblotting was performed using the same primary and secondary antibodies as in IHC (diluted 1:200 and 1:2000, respectively) followed by incubation with avidin-biotin-peroxidase complex (Vectastain ABC kit, Vector Laboratories, Burlingame, CA, USA). In order to check for nonspecific binding of secondary antibodies, primary antibodies were omitted in control samples. Immunoreactive proteins were visualized using an ECL chemiluminescence kit (Pierce Biotechology, Rockford, IL, USA) followed by autoradiography (Kodak MXB film and developing reagents, Paris, France). Molecular mass markers were from BioRad Laboratories (Hertfordshire, UK).

\subsection{Immunoprecipitation}

Thyroid tissue protein extracts were prepared as described for Western blot, with the exception that two different conditions were used for extraction: nondenaturing (lysis buffer: $20 \mathrm{mM}$ Tris $\mathrm{HCl} \mathrm{pH} \mathrm{8,} 137 \mathrm{mM}$ $\mathrm{NaCl}, 10 \%$ glycerol, 1\% NP40, 2 mM EDTA) and denaturing (5\% SDS). Approximately $2 \mathrm{mg}$ of proteins (diluted with lysis buffer to the final volume of $500 \mu \mathrm{l}$ ) were used per sample. To prevent nonspecific binding, the lysates were pre-cleared with $30 \mu \mathrm{l}$ of bead slurry for $30 \mathrm{~min}$ at $4^{\circ} \mathrm{C}$, and after centrifugation the bead pellet was discarded and supernatant was kept for immunoprecipitation.

Bead slurry $(20 \mu \mathrm{l})$ of Protein A immobilized on agarose (Fluka 82486, Sigma-Aldrich, St. Louis, MO, USA) was washed thrice with lysis buffer and incubated with $2 \mu \mathrm{g}$ of sc894 caveolin-1 antibody (Santa Cruz Biotechnology, Santa Cruz, CA, USA) in $500 \mu 1$ lysis buffer, overnight at $4^{\circ} \mathrm{C}$ with agitation. After washing three times with lysis buffer, the beads were incubated with thyroid tissue protein extracts for $6 \mathrm{~h}$ at $4{ }^{\circ} \mathrm{C}$ on a rotating wheel. After centrifugation, the bead pellet was washed thrice with lysis buffer. As a control, protein A agarose without antibody coupled to it was also incubated with the same amount of lysate. Immunoprecipitated caveolin-1 was recovered by resuspending the beads in Laemmli sample buffer. Western blot analysis was performed using AV09019 caveolin-1 antibody (Sigma-Aldrich, St Louis, MO, USA).

\subsection{Statistical analysis}

The results were analyzed statistically with SPSS 12.0.1 (SPSS Inc., Chicago, IL, USA) for Windows. The Mann-Whitney U test was used for comparison between groups and $p<0.05$ was considered significant. Receiver operating characteristic (ROC) curve analysis was performed to establish cut-off values for the antibodies used in immunohistochemistry and to determine their diagnostic potential. We constructed the ROC curves using sensitivity and specificity calculated for each score for both antibodies. The cut-off was set at the score that showed both maximum sensitivity and specificity, that is the point located closest to the point $(0,1)$ on the graph. Optimal cut-off values for both markers were set at the score 1 i.e. the groups were classified as marker-negative (score 0) and marker-positive (scores 1-3). The area under the ROC curve (AUC) in ROC analysis was used to evaluate the ability of an antibody to discriminate between thyroid histological counterparts, thus providing information about the diagnostic accuracy of each marker. In addition, positive and negative predictive values and diagnostic accuracy were calculated after the cut-off was introduced.

The Spearman correlation coefficient was used to determine the degree of correlation between the two markers and their association with clinicopathological data. After the appropriate cut-off values were introduced (cut-off at score 1 for sc894 producing negative and positive groups, and cut-off at score 2 for AV09019 antibody, dividing the cases into low and high groups), the association of immunohistochemical examinations and clinicopathological data was compared using 2tailed Fisher's exact test.

\section{Results}

\subsection{Caveolin-1 immunohistochemistry}

Paraffin-embedded tissue samples from patients with differentiated thyroid tumors were examined immuno- 
Table 1

Immunohistochemical expression of caveolin-1 detected with antibodies sc894 (Santa Cruz) and AV09019 (Sigma) in different thyroid lesions

\begin{tabular}{lllrrrr}
\hline & Histology & \multicolumn{3}{c}{ Expression grade } & Total \\
\cline { 2 - 7 } sc894 Santa Cruz & & 0 & 1 & 2 & 3 & 111 \\
\cline { 2 - 7 } antibody & Malignant & & & & & \\
& clPTC & $1(5.6 \%)$ & 7 & 7 & 3 & 18 \\
& fvPTC & $7(38.9 \%)$ & 7 & 3 & 1 & 18 \\
& FTC & $8(61.5 \%)$ & 3 & 2 & 0 & 13 \\
& Nonmalignant & & & & \\
& FTA & $17(85 \%)$ & 3 & 0 & 0 & 20 \\
AV09019 Sigma & PT & $23(54.8 \%)$ & 13 & 5 & 1 & 42 \\
\cline { 2 - 7 } antibody & Histology & Expression grade & & Total \\
\cline { 2 - 7 } & Malignant & & 1 & 2 & 3 & 178 \\
\hline & clPTC & $2(6.1 \%)$ & 6 & 8 & 17 & 33 \\
& fvPTC & $5(14.7 \%)$ & 11 & 11 & 7 & 34 \\
& FTC & $9(47.4 \%)$ & 5 & 4 & 1 & 19 \\
& Nonmalignant & & & & \\
& FTA & $25(83.3 \%)$ & 3 & 1 & 1 & 30 \\
& PT & $51(82.2 \%)$ & 8 & 1 & 2 & 62 \\
\hline
\end{tabular}

Staining categories: 0 - absence of staining or focal staining, 1 weak, 2 - moderate and 3 - strong diffuse cytoplasmic staining of thyrocytes.

Histology: clPTC-classical variant of papillary thyroid carcinoma, fvPTC-follicular variant of papillary thyroid carcinoma, FTCfollicular thyroid carcinoma, FTA-follicular thyroid adenoma, PTperitumoral tissue. Comparison between groups by the MannWhitney U test: Using Santa Cruz Ab, $p<0.05$ for clPTC vs. fvPTC, fvPTC vs. FTA, and FTA vs. PT. Using Sigma Ab, $p<$ 0.05 for clPTC vs. fvPTC, fvPTC vs. FTC, fvPTC vs. FTA and FTC vs. FTA. Values in parentheses are percentages of negatively stained cells.

histochemically, using two commercial polyclonal antibodies against caveolin-1. Figure 1 shows representative examples of caveolin-1 immunostaining with sc894 (Santa Cruz) and AV09019 (Sigma) antibody on thyroid tissue samples. The staining scores for individual samples are presented in Table 1.

With sc894 antibody, $67.3 \%$ (33/49) of malignant and $35.5 \%(22 / 62)$ of nonmalignant samples showed immunoreactivity for caveolin-1 with a cut-off value of score 1 . The classical variant of PTC was positive in all but one case $(17 / 18 ; 94.4 \%)$, while high caveolin- 1 immunostaining (score 2 or 3 ) was seen in more than half of the cases $(55.5 \%)$. FvPTC showed immunoreactivity in $11 / 18(61.1 \%)$ cases, and the difference in immunohistochemical staining scores between the groups of clPTC and fvPTC was statistically significant $(p<$ 0.05 , Mann Whitney $\mathrm{U}$ test). FTC was positive in 5 out of $13(38.5 \%)$ samples; nevertheless, compared to fvPTC, immunoreactivity was not significantly different between the two groups.

Regarding nonmalignant tissue, caveolin-1 cytoplasmic staining was negative in 17 out of $20(85 \%)$ FTA and in 23 out of $42(54.8 \%)$ specimens of peritumoral tissues. The difference in staining between these two groups was significant ( $p<0.05$ for FTA vs. PT). A statistically significant difference was also observed between FTA and fvPTC. Intensive staining of fibroblasts and vascular endothelial cells with sc894 antibody was observed in all cases, regardless of the tumor type.

Immunostaining of caveolin-1 with AV09019 antibody was visible in $81.4 \%$ cases of malignant tumors, and $17.4 \%$ of nonmalignant samples. Classical PTC was mostly positive $(31 / 33 ; 93.9 \%)$, with $75.8 \%$ of high-scoring cases (score 2 or 3 ). The follicular variant of PTC was also positive in most cases $(85.3 \%)$, while FTC were positive in 10/19 (52.6\%) samples. Both FTA and PT were usually negative $(83.3 \%$ and $82.2 \%$ respectively). Statistically significant differences in immunohistochemical scores were observed between the following groups: clPTC vs. fvPTC, fvPTC vs. FTC, fvPTC vs. FTA and FTC vs. FTA $(p<0.05$, Mann Whitney U test). Weak immunoreactivity was observed in fibroblasts and vascular endothelial cells.

\subsection{Diagnostic performance of sc894 and AV09019 antibodies}

In order to determine which antibody is a better marker for differential diagnostics, we calculated the sensitivity and specificity for each one and performed ROC analysis using the cut-off score 1 , to evaluate the diagnostic accuracy (Table 2). The results obtained indicate that immunostaining with sc894 antibody could be used to differentiate between FTA and fvPTC, and also between PTC and FTC. Since overall discrimination is a function of the AUC, positive staining for caveolin-1, using the established cut-off value was a fair identifier of PTC. In contrast to sc894, which gave no statistically significant difference between FTA and FTC, the AV09019 antibody showed potential to differentiate FTA and FTC with a diagnostic accuracy of $71.4 \%(p=0.035)$.

Follicular adenoma and the follicular variant of papillary carcinoma (FTA vs. fvPTC) were distinguished using AV09019 antibody, with high sensitivity $(85.3 \%)$ and specificity $(83.3 \%)$ and area under the curve $(\mathrm{AUC}=0.843, p<0.001)$. More importantly, this antibody detected statistically significant caveolin1 expression in FTC when compared to fvPTC ( $p=$ $0.05)$.

Expression of caveolin-1 was significantly more frequent in classical than in the follicular variant of PTC. However, when the cut-off point was introduced, ROC 


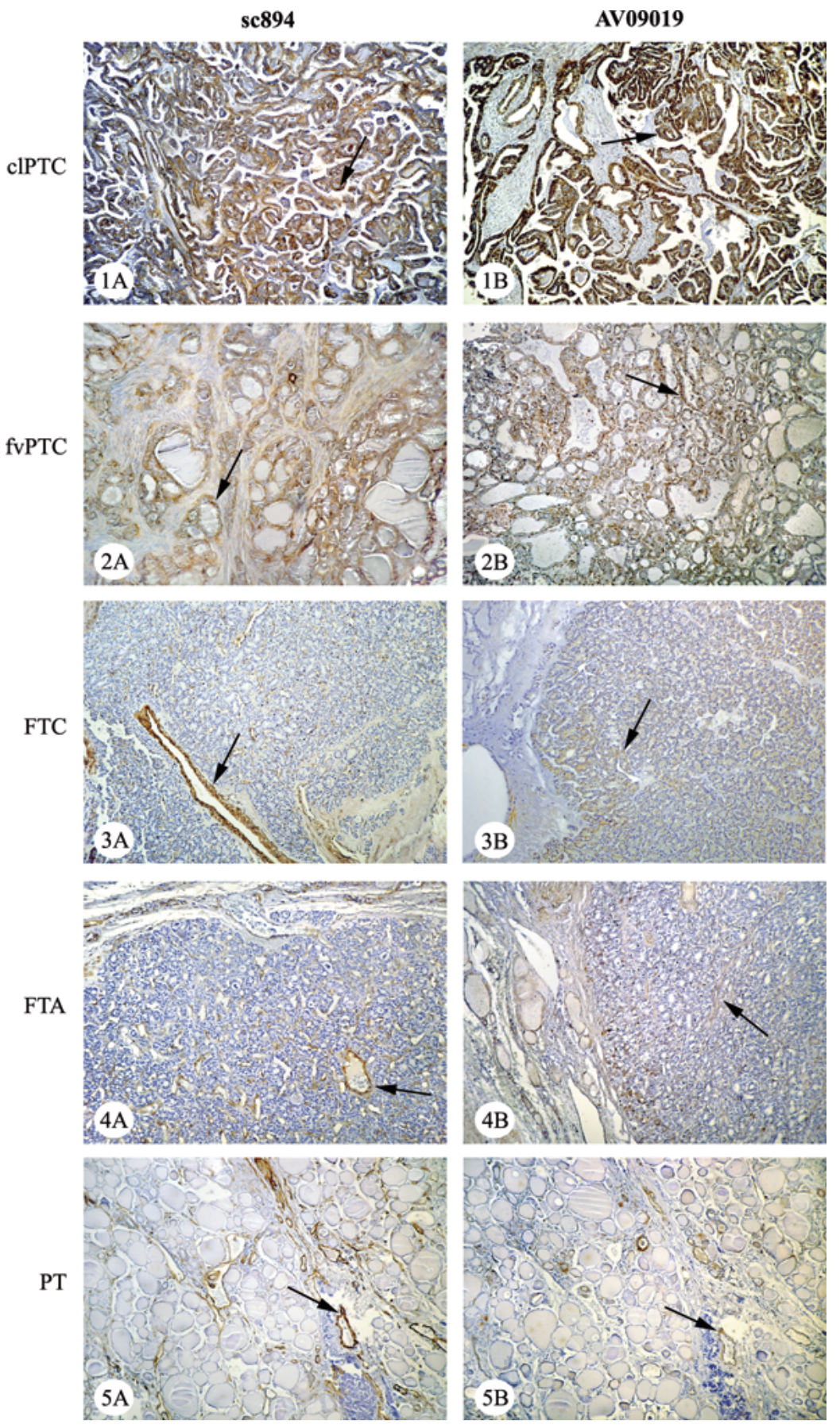

Fig. 1. Representative micrographs of caveolin-1 immunohistochemical staining in thyroid tumors and adjacent peritumoral tissue. Black arrows indicate caveolin-1 positive staining. Caveolin-1 staining in clPTC was strong in cytoplasm and membrane of carcinoma cells when sc894 antibody was used (1A) and also when AV09019 antibody was used (1B). Moderate caveolin-1 expression in fvPTC was detected with sc894 (2A) and AV09019 (2B). Follicular thyroid carcinoma cells were negative for caveolin-1 with sc894, while vascular endothelia stained strongly (3A); however, with AV09019 antibody we observed weak staining of thyrocytes in FTC (3B). The staining of follicular epithelial cells in adenomas was negative with both antibodies (4A and 4B), but positive in endothelial cells (4A) and stromal cells (4B). Follicular epithelial cells in peritumoral tissue were also immunonegative with both antibodies, with the exception that sc894 stained endothelial cells strongly ( $5 \mathrm{~A}$ ) and AV09019 moderately (5B). Original magnification was 10x in all micrographs. (Colours are visible in the online version of the article; http://dx.doi.org/10.3233/DMA-2012-00934) 
Table 2

Diagnostic performance of sc894 and AV09019 antibody, calculated by ROC curve analysis

\begin{tabular}{|c|c|c|c|c|c|c|c|c|c|c|c|}
\hline \multirow{10}{*}{$\begin{array}{l}\text { sc894 Santa Cruz } \\
\text { antibody }\end{array}$} & Compared tissue & AUC & $\mathrm{p}$ & Cut-off & $\mathrm{AUC}(\mathrm{co})$ & $\mathrm{p}$ & Sens. & Spec. & PPV & NPV & $\mathrm{ACC}$ \\
\hline & FTA vs. PT & 0.662 & 0.041 & 1 & 0.651 & n.s. & 1 & 1 & 1 & 1 & 1 \\
\hline & FTA vs. FTC & 0.629 & n.s. & l & l & l & I & I & I & I & l \\
\hline & fvPTC vs. FTA & 0.747 & 0.009 & 1 & 0.731 & 0.015 & $61.1 \%$ & $85.0 \%$ & $78.5 \%$ & $70.8 \%$ & $73.6 \%$ \\
\hline & fvPTC vs. FTC & 0.613 & n.s. & l & I & I & 1 & I & I & I & 1 \\
\hline & fvPTC vs. clPTC & 0.735 & 0.016 & 1 & 0.667 & n.s. & I & I & l & I & l \\
\hline & PTC vs. FTA & 0.843 & $<0.001$ & 1 & 0.814 & $<0.001$ & $77.8 \%$ & $85 \%$ & $90.3 \%$ & $68 \%$ & $80.4 \%$ \\
\hline & PTC vs. FTC & 0.720 & 0.020 & 1 & 0.697 & 0.037 & $77.8 \%$ & $61.5 \%$ & $84.8 \%$ & $50.0 \%$ & $73.5 \%$ \\
\hline & PTC vs. Nonmalignant & 0.745 & $<0.001$ & 1 & 0.711 & 0.001 & $77.8 \%$ & $64.5 \%$ & $56.0 \%$ & $83.3 \%$ & $69.4 \%$ \\
\hline & Malignant vs. Nonmalignant & 0.681 & 0.001 & 1 & 0.659 & 0.004 & $67.3 \%$ & $64.5 \%$ & $60.0 \%$ & $71.4 \%$ & $65.8 \%$ \\
\hline \multirow{10}{*}{$\begin{array}{l}\text { AV09019 Sigma } \\
\text { antibody }\end{array}$} & Compared tissue & AUC & $\mathrm{p}$ & Cut-off & $\mathrm{AUC}(\mathrm{co})$ & $\mathrm{p}$ & Sens. & Spec. & PPV & NPV & $\mathrm{ACC}$ \\
\hline & FTA vs. PT & 0.502 & n.s. & I & l & / & 1 & l & I & 1 & l \\
\hline & FTA vs. FTC & 0.682 & 0.034 & 1 & 0.680 & 0.035 & $52.6 \%$ & $83.3 \%$ & $66.6 \%$ & $73.5 \%$ & $71.4 \%$ \\
\hline & fvPTC vs. FTA & 0.857 & $<0.001$ & 1 & 0.843 & $<0.001$ & $85.3 \%$ & $83.3 \%$ & $85.2 \%$ & $83.3 \%$ & $84.3 \%$ \\
\hline & fvPTC vs. FTC & 0.704 & 0.015 & 1 & 0.663 & 0.050 & $85.2 \%$ & $47.3 \%$ & $74.3 \%$ & $64.2 \%$ & $71.6 \%$ \\
\hline & fv-PTC vs. clPTC & 0.676 & 0.013 & 1 & 0.543 & n.s. & / & I & l & I & l \\
\hline & PTC vs. FTA & 0.889 & $<0.001$ & 1 & 0.864 & $<0.001$ & $89.6 \%$ & $83.3 \%$ & $92.3 \%$ & $78.1 \%$ & $87.6 \%$ \\
\hline & PTC vs. FTC & 0.766 & $<0.001$ & 1 & 0.685 & 0.014 & $89.5 \%$ & $47.4 \%$ & $85.7 \%$ & $56.2 \%$ & $80.2 \%$ \\
\hline & PTC vs. Nonmalignant & 0.892 & $<0.001$ & 1 & 0.861 & $<0.001$ & $89.5 \%$ & $82.6 \%$ & $78.9 \%$ & $91.6 \%$ & $85.5 \%$ \\
\hline & Malignant vs. Nonmalignant & 0.845 & $<0.001$ & 1 & 0.820 & $<0.001$ & $81.4 \%$ & $82.6 \%$ & $81.4 \%$ & $82.6 \%$ & $82.0 \%$ \\
\hline
\end{tabular}

Nonmalignant tissue comprises FTA and PT; AUC: area under the ROC curve; $p<0.05$ considered significant; Cut-off: score 1 was selected as the threshold value; AUC(co): area under the ROC curve after the cut-off was introduced; Sens.: sensitivity; Spec.: specificity, PPV: positive predictive value; NPV: negative predictive value; ACC: diagnostic accuracy; n.s.: not significant. Bolded values represent AUC with good diagnostic potential.

curve analysis did not confirm this. Thus, irrespective of the antibody used, expression of caveolin-1 could not distinguish classical from the follicular variant of PTC.

It is important to emphasize that even though AV09019 was also more sensitive than sc894 (89.5\% and $77.8 \%$, respectively) for the differential diagnosis of PTC from FTC, the more recently available antibody was less specific. Overall performance was moderate for both antibodies and did not exceed an average AUC of 0.7 , but positive predictive value (precision) for sc894 and AV09019 was favorable $(84.8 \%$ and $85.7 \%$, respectively). The diagnostic accuracy of AV09019 was greater than sc894 in discriminating total papillary carcinoma from nonmalignant tissue (ACC values $85.5 \%, p<0.001$ for AV09019 and 69.4\%, $p=$ 0.001 for sc894), as well as malignant tumors from nonmalignant tissue (ACC values $82.0 \%, p<0.001$ for AV09019 and $65.8 \%, p=0.004$ for sc894). It is also important to emphasize that for the aforementioned tissue types, AV09019 had higher sensitivity, specificity, positive predictive value and negative predictive value than sc894, (Table 2).

\subsection{Western blot of caveolin-1 expression in thyroid tumor and peritumoral tissue}

The expression of caveolin-1 in papillary thyroid carcinoma (PTC) and matched non-tumor tissue was ana- lyzed by immunohistochemistry and by WB. Western blot displayed intensive bands at the expected molecular weight of caveolin-1 (22 kDa) with sc894 antibody (Fig. 2A). Both WB analysis and immunohistochemical staining with sc894 antibody demonstrated no striking difference between caveolin-1 expression in tumor and matched non-tumor tissue. On the other hand, WB analysis with AV09019 antibody (Fig. 2B) revealed a protein band of about $22 \mathrm{kDa}$ in analyzed tumor tissue extracts and no signal in peritumoral tissue, although the background signal was high. Figure $2 \mathrm{~B}$ also shows positive caveolin-1 staining in tumor tissue, and negative staining in matched non-tumor tissue.

Due to the discrepancy of results obtained with these two antibodies, we decided to test whether they recognize the same protein. After immunoprecipitation using sc894 antibody, we probed the Western blots with AV09019 antibody (Fig. 2C). The results confirmed the presence of a $22 \mathrm{kDa}$ band in the samples where sc894 antibody was coupled to agarose in contrast to those where no antibody was used. Two different conditions were employed for protein isolation from thyroid tissue: denaturating and non-denaturating, and both gave the same result.

\subsection{Caveolin-1 expression correlates with extrathyroidal extension}

After examining the expression pattern of caveolin1 , we aimed to analyze the association of caveolin-1 


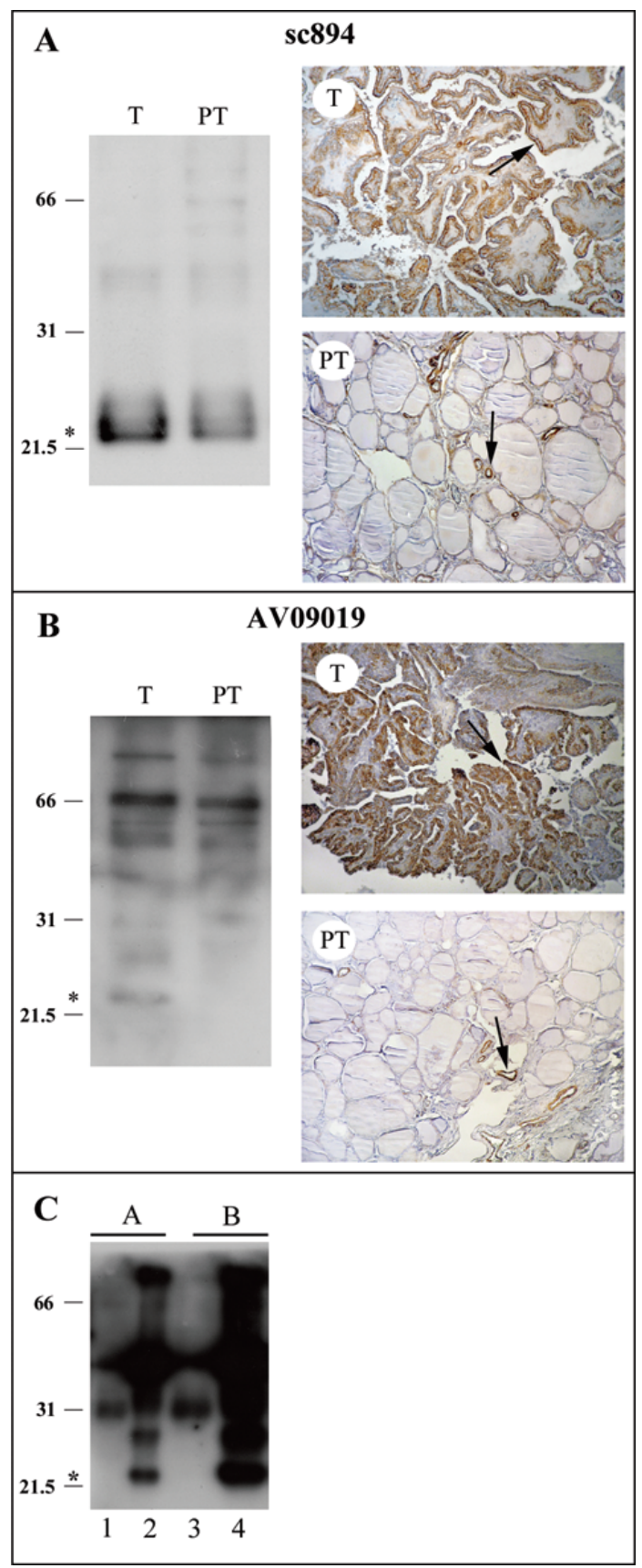

Fig. 2. Caveolin-1 expression analyzed by immunohistochemistry and Western blot using sc894 and AV09019 antibody, in tumor (T) and matched non-tumor tissue i.e. peritumoral tissue (PT) from the same patient. Black arrows indicate caveolin-1 positive staining. Original magnification was 10x in all micrographs. (A) sc894 staining detected bands at a molecular weight corresponding to caveolin-1 in both tumor and peritumoral tissue (asterisk). In immunohistochemistry, thyrocytes of tumoral tissue i.e. papillary thyroid carcinoma were stained moderately, while thyroid follicular cells in peritumoral tissue were not stained or stained weakly. Vascular endothelia were stained intensely. (B) AV09019 antibody revealed a band at the expected molecular weight only in tumoral tissue (asterisk). Immunostaining was strong in thyrocytes of papillary thyroid carcinoma and negative in peritumoral tissue of thyroid follicular cells, but positive in vascular endothelial cells. (C) Immunoprecipitation of caveolin-1 using sc894 antibody. Thyroid tissue proteins were extracted with non-denaturating (A) and denaturating (B) lysis buffer. The extracts were subjected to immunoprecipitation with protein A agarose beads. Lanes 1 and 3 correspond to immunoprecipitates with "empty" beads and lanes 2 and 4 with beads coupled to caveolin- 1 sc894 antibody. The asterisk denotes the specific band detected by AV09019 antibody corresponding to the caveolin-1 Mw. (Colours are visible in the online version of the article; http://dx.doi.org/10.3233/DMA-2012-00934) 
Table 3

Correlation between clinicopathological parameters and immunohistochemical caveolin-1 expression for antibodies sc894 and AV09019 in PTC

\begin{tabular}{|c|c|c|c|c|c|}
\hline Spearman's rho & $\begin{array}{l}\text { AV09019 Sigma } \\
\text { antibody }\end{array}$ & $\begin{array}{l}\mathrm{pT} \\
\text { status }\end{array}$ & $\overline{\mathrm{LNM}}$ & ID & $\mathrm{Ei}$ \\
\hline \multicolumn{6}{|l|}{ sc894 Santa Cruz antibody } \\
\hline Correlation coefficient & $0.693^{* *}$ & 0.303 & 0.041 & -0.065 & $0.351^{*}$ \\
\hline Significance & $<\mathbf{0 . 0 0 1}$ & 0.077 & 0.814 & 0.742 & 0.036 \\
\hline $\mathrm{N}$ & 112 & 35 & 36 & 28 & 36 \\
\hline \multicolumn{6}{|l|}{ AV09019 Sigma antibody } \\
\hline Correlation coefficient & & $0.275^{*}$ & 0.220 & -0.069 & $0.394^{* *}$ \\
\hline Significance & & $\mathbf{0 . 0 3 0}$ & 0.078 & 0.726 & 0.001 \\
\hline $\mathrm{N}$ & & 62 & 65 & 28 & 65 \\
\hline
\end{tabular}

$\mathrm{N}$ - number of pairs of variables; pT status - pathological $\mathrm{T}$ status (T category of TNM staging); LNM lymph node metastases; Ei - extrathyroidal invasion; ID - intraglandular dissemination; bolded values represent significant correlations between parameters.

${ }^{*}$ correlation significant at the 0.05 level (2-tailed); ${ }^{* *}$ correlation significant at the 0.01 level (2-tailed).

Table 4

Correlation between immunohistochemical expression of caveolin-1 and clinicopathological findings for PTC

\begin{tabular}{|c|c|c|c|c|c|c|c|c|}
\hline \multirow[t]{2}{*}{ Parameters } & \multicolumn{4}{|c|}{ sc894 Santa Cruz } & \multicolumn{4}{|c|}{ AV09019 Sigma } \\
\hline & Total & Negative & Positive & $p$ & Total & Low & High & $p$ \\
\hline pT status & 35 & & & 0.662 & 62 & & & 0.213 \\
\hline $\mathrm{T} 1$ & 5 & 1 & 4 & & 10 & 5 & 5 & \\
\hline $\mathrm{T} 2$ & 21 & 5 & 16 & & 29 & 9 & 20 & \\
\hline $\mathrm{T} 3$ & 5 & 2 & 3 & & 10 & 5 & 5 & \\
\hline $\mathrm{T} 4$ & 4 & 0 & 4 & & 13 & 2 & 11 & \\
\hline Nodular status & 36 & & & 1.000 & 65 & & & 0.084 \\
\hline Absent & 31 & 7 & 24 & & 55 & 22 & 33 & \\
\hline Present & 5 & 1 & 4 & & 10 & 1 & 9 & \\
\hline Intraglandular & & & & 1.000 & & & & 1.000 \\
\hline dissemination & 28 & & & & 28 & & & \\
\hline Absent & 21 & 5 & 16 & & 21 & 7 & 14 & \\
\hline Present & 7 & 3 & 5 & & 7 & 2 & 5 & \\
\hline Extrathyroid invasion & 36 & & & 0.076 & 65 & & & 0.009 \\
\hline Absent & 26 & 8 & 18 & & 46 & 21 & 25 & \\
\hline Present & 10 & $\mathbf{0}$ & 10 & & 19 & 2 & 17 & \\
\hline
\end{tabular}

Caveolin-1 staining (sc894 Santa Cruz antibody): negative, score 0; positive, score 1, 2 and 3. Caveolin-1 staining (AV09019 Sigma antibody): low, score 0 and 1; high, score 2 and 3. pT status - pathological T status (T category of TNM staging); bolded values represent significant correlations. Results analyzed by Fisher exact test.

expression with clinicopathological features of papillary thyroid carcinomas, using several correlation tests. Spearman's analysis (Table 3) revealed a positive correlation between sc894 and AV09019 antibody ( $\rho=$ $0.693, p<0.001$ ). For both antibodies, we observed a significant positive correlation of caveolin-1 expression and extrathyroidal invasion of PTC ( $\rho=0.351, p=$ 0.036; $\rho=0.394, p=0.001$ for sc894 and AV09019, respectively). Additionally, using AV09019 antibody caveolin-1 expression positively correlated with pathological T status ( $\rho=0.275, p=0.03$ ). When we introduced cut-off score 1 for sc894 antibody, based on the criteria described in Materials and Meterial (Table 4), no significant correlation between caveolin-1 expression and clinicopathological parameters was found. For AV09019 antibody, the cut-off score was set even high- er, at the value 2 , i.e. cases were divided into low and high caveolin-1 expression groups. High caveolin-1 expression was also found to be significantly correlated with extrathyroidal invasion $(p=0.009)$.

\section{Discussion}

In the present study, caveolin-1 expression was evaluated in the human thyroid neoplasia spectrum (FTA, FTC, PTC), as well as in adjacent peritumoral tissue with the aim of comprehensively examining differences of expression detected by two antibodies against cav-1, and secondly, to investigate the association of caveolin1 expression levels with aggressive PTC. Two commercially available antibodies were selected: sc894 an- 
tibody (Santa Cruz) used previously by Ito and colleagues [6,21], and AV09019 (Sigma) antibody. To the best of our knowledge, the latter antibody has not been previously used for immunohistochemical staining of thyroid neoplastic tissue.

In agreement with Ito et al. [6,21], we found that caveolin-1 was generally overexpressed in the papillary thyroid carcinoma group as a whole (classic and follicular variant) when compared to normal tissue, while this protein was mainly negative in FTC and FTA. Aldred et al. [5] reported down-regulation of the caveolin1 gene in FTC but, on the other hand, there was no change in caveolin-1 expression in PTC in comparison to normal thyroid tissue. In our study, immunohistochemical analysis of PTCs using sc894 antibody, gave positive results in a large majority of cases $(94.4 \%$ for clPTC and $61.1 \%$ for fvPTC). This is somewhat different from the $67.1 \%$ reported by Ito et al. [21] for PTC. In addition, our study showed caveolin-1 positivity in a larger proportion of analyzed FTC cases (38.5\%). Furthermore, in our hands, sc894 antibody was not helpful in the fvPTC/ FTC differential diagnostics as already reported by Ito et al. [6]. This disagreement in findings obtained using the same commercial antibody can be attributed to different factors, including the underpowered series, or the use of different processing techniques and cut-off values in immunohistochemical analysis, among others.

According to our results, the new commercially available antibody against caveolin-1 has higher diagnostic accuracy than sc894 antibody for distinguishing PTC from non-malignant tissues (ACC value $85.5 \%$ for AV09019 and 69.4\% for sc894). Moreover, AV09019 was positive in $37.5 \%$ cases of sc894 negative PTCs, whereas there were no sc894 positive cases in AV09019 negative cancers. The new AV09019 antibody also gave positive staining in $52.6 \%$ FTCs and $16.7 \%$ FTAs. Nevertheless, immunohistochemical results obtained with this antibody showed statistically significant differences between: FTC, fvPTC and FTA. Thus, the immunohistochemical analysis using AV09019 antibody had better discriminatory power in the differential diagnosis of almost all analyzed histotypes in comparison to that of sc894. Even though AV09019 was also more sensitive than sc894 for the differential diagnosis of PTC from FTC, it was less specific. Thus, overall performance was moderate for both antibodies and did not exceed an average AUC of 0.7. However, with $84.8 \%$ positive predictive value (precision) for sc894 and $85.7 \%$ for AV09019, both antibodies were helpful discriminators between PTC and FTC.
We found differences in the immunohistochemical staining patterns of these two markers within thyroid tissue. The Sigma antibody against caveolin-1, AV09019, gave more intense cytoplasmic staining in epithelial cells and lower staining in fibroblasts and endothelial cells in comparison to sc894 antibody. Nevertheless, both polyclonal antibodies showed a significant positive correlation in immunohistochemical staining scores in thyrocytes of the analyzed specimens, with discrepant scores in only $9 \%$ of cases. This result is consistent with our findings for immunoprecipitation i.e. both antibodies detect the same protein corresponding to caveolin-1. In addition, Western blot analysis showed distinct and specific detection of caveolin-1 protein in thyroid tissue extracts using sc894 antibody, but less effective detection with AV09019 antibody. Thus, although not as effective in Western blot as sc894, AV09019 was superior to sc894 in immunohistochemistry.

We also studied the relation of caveolin-1 to clinicopathological parameters of papillary thyroid carcinoma by correlating the results of immunohistochemical staining with available clinicopathological data. According to our findings, expression of caveolin-1 was positively correlated with extrathyroidal invasion regardless of the antibody employed. Moreover, using AV09019 a correlation was found with advanced pathological $\mathrm{T}$ status. This indicates that there is a link between caveolin-1 expression and aggressive behavior of papillary thyroid carcinoma. Regarding this issue, Kim et al. [23] recently observed that the expression status of caveolin-1 in thyrocytes was not significantly correlated with any features related to the aggressiveness of thyroid cancers of different histological types (papillary thyroid carcinoma, diffuse sclerosing variant of papillary carcinoma and anaplastic thyroid carcinoma). A plausible explanation for this discrepancy could be the different caveolin-1 antibody they used, and the fact that, when analyzing the performance of caveolin1 , the authors grouped together thyroid cancers of different histological types, which are well known to have very different clinical and molecular characteristics.

For other cancer types there is a solid amount of evidence in the literature confirming correlation of caveolin-1 upregulation with poor clinical prognosis. For example, in prostate cancer, positive caveolin-1 expression was associated with clinical markers of disease progression, such as extraprostatic extension, seminal vesicle invasion and lymph node involvement [24]. Similarly, overexpression of this protein was detected in deceased and metastatic breast cancer patients [25]. 
Correlation between caveolin-1 intensity and tumor size, TNM stage and metastasis was confirmed in a renal carcinoma [26]. On the other hand, a literature search revealed examples of the opposite effect, that is, a beneficial effect of caveolin-1 upregulation on cancer prognosis. In salivary gland tumors, downregulated caveolin-1 expression was associated with advanced clinical stage and development of recurrent disease, indicating a poor prognosis [15]. In bile duct carcinoma, caveolin-1 immunostaining negatively correlated with patient age and T stage [14]. Thus, caveolin-1 expression can be positively or negatively correlated with tumor progression in different types of cancer. Our data display correlation of caveolin-1 expression with aggressiveness of papillary thyroid cancer, suggesting that the immunostaining pattern detected by each antibody against cav-1 may increase the ability of clinicians to evaluate the progression of PTC patients.

To summarize, the new commercially available antibody against caveolin-1, AV09019 is superior to sc894 antibody in immunohistochemistry but not as effective in Western blot. Regarding their application as immunohistochemical diagnostic markers, both caveolin1 antibodies could be molecular adjunct tools for differentiating some histologic subtypes of thyroid tumors. In addition, increased expression of caveolin-1 protein is positively related to extrathyroidal invasion of PTC and may be considered as an indicator of papillary carcinoma progression. However, to derive unequivocal diagnostic or prognostic information by immunohistochemistry on caveolin-1, further monitoring of this caveola protein is essential.

\section{Acknowledgments}

This work was supported by the Ministry of Education and Science of the Republic of Serbia, project 173050: "Molecular characterization of thyroid gland tumors: biological and clinical aspects".

We thank Professor Marija Havelka and Professor Svetislav Tatic for help in revising the histological slides. The authors wish to express their gratitude to Dr. Anna Nikolic, for language correction of the manuscript.

\section{References}

[1] A.Y. Chen, A. Jemal and E.M. Ward, Increasing incidence of differentiated thyroid cancer in the United States, 1988-2005, Cancer 115 (2009), 3801-3807.
[2] L. Davies and H.G. Welch, Increasing incidence of thyroid cancer in the United States, 1973-2002, JAMA 295 (2006), 2164-2167.

[3] O. Olaleye, U. Ekrikpo, R. Moorthy, O. Lyne, J. Wiseberg, M. Black and D. Mitchell, Increasing incidence of differentiated thyroid cancer in South East England: 1987-2006, Eur Arch Otorhinolaryngol 268 (2011), 899-906.

[4] S.J. Schonfeld, C. Lee and A. Berrington de Gonzalez, Medical exposure to radiation and thyroid cancer, Clin Oncol $(R$ Coll Radiol) 23 (2011), 244-250.

[5] M.A. Aldred, Y. Huang, S. Liyanarachchi, N.S. Pellegata, O. Gimm, S. Jhiang, R.V. Davuluri, A. de la Chapelle and C. Eng, Papillary and follicular thyroid carcinomas show distinctly different microarray expression profiles and can be distinguished by a minimum of five genes, J Clin Oncol 22 (2004), 3531-3539.

[6] Y. Ito, H. Yoshida, C. Tomoda, T. Uruno, Y. Takamura, A. Miya, K. Kobayashi, F. Matsuzuka, Y. Nakamura, K. Kakudo, K. Kuma and A. Miyauchi, Caveolin-1 and 14-3-3 sigma expression in follicular variant of thyroid papillary carcinoma, Pathol Res Pract 201 (2005), 545-549.

[7] R.G. Parton and K. Simons, The multiple faces of caveolae, Nat Rev Mol Cell Biol 8 (2007), 185-194.

[8] T. Okamoto, A. Schlegel, P.E. Scherer and M.P. Lisanti, Caveolins, a family of scaffolding proteins for organizing "preassembled signaling complexes" at the plasma membrane, $J$ Biol Chem 273 (1998), 5419-5422.

[9] A.W. Cohen, R. Hnasko, W. Schubert and M.P. Lisanti, Role of caveolae and caveolins in health and disease, Physiol Rev 84 (2004), 1341-1379.

[10] M. Bastiani and R.G. Parton, Caveolae at a glance, J Cell Sci 123 (2010), 3831-3836.

[11] A.F. Quest, J.L. Gutierrez-Pajares and V.A. Torres, Caveolin1: an ambiguous partner in cell signalling and cancer, $J$ Cell Mol Med 12 (2008), 1130-1150.

[12] C. Boscher and I.R. Nabi, Caveolin-1: role in cell signaling, Adv Exp Med Biol 729 (2012), 29-50.

[13] T.M. Williams and M.P. Lisanti, Caveolin-1 in oncogenic transformation, cancer, and metastasis, Am J Physiol Cell Physiol 288 (2005), C494-506.

[14] S. Murakami, M. Miyamoto, Y. Hida, Y. Cho, A. Fukunaga, T. Oshikiri, K. Kato, T. Kurokawa, M. Suzuoki, Y. Nakakubo, K. Hiraoka, T. Itoh, T. Shinohara, T. Morikawa, O. Okushiba, S. Kondo and H. Katoh, Caveolin-I overexpression is a favourable prognostic factor for patients with extrahepatic bile duct carcinoma, Br J Cancer 88 (2003), 1234-1238.

[15] L. Shi, X.M. Chen, L. Wang, L. Zhang and Z. Chen, Expression of caveolin-1 in mucoepidermoid carcinoma of the salivary glands: correlation with vascular endothelial growth factor, microvessel density, and clinical outcome, Cancer 109 (2007), 1523-1531.

[16] J. Cui, L.R. Rohr, G. Swanson, V.O. Speights, T. Maxwell and A.R. Brothman, Hypermethylation of the caveolin-1 gene promoter in prostate cancer, Prostate 46 (2001), 249-256.

[17] J.A. Engelman, X.L. Zhang and M.P. Lisanti, Sequence and detailed organization of the human caveolin -1 and -2 genes located near the D7S522 locus (7q31.1). Methylation of a CpG island in the 5' promoter region of the caveolin-1 gene in human breast cancer cell lines, FEBS Lett 448 (1999), 221230.

[18] I. Tamaskar and M. Zhou, Clinical implications of caveolins in malignancy and their potential as therapeutic targets, Curr Oncol Rep 10 (2008), 101-106. 
[19] E. Burgermeister, M. Liscovitch, C. Rocken, R.M. Schmid and M.P. Ebert, Caveats of caveolin-1 in cancer progression, Cancer Lett 268 (2008), 187-201.

[20] M.J. Costa, Y. Song, P. Macours, C. Massart, M.C. Many, S. Costagliola, J.E. Dumont, J. Van Sande and V. Vanvooren, Sphingolipid-cholesterol domains (lipid rafts) in normal human and dog thyroid follicular cells are not involved in thyrotropin receptor signaling, Endocrinology 145 (2004), 14641472 .

[21] Y. Ito, H. Yoshida, K. Nakano, K. Kobayashi, T. Yokozawa, K. Hirai, F. Matsuzuka, N. Matsuura, K. Kakudo, K. Kuma and A. Miyauchi, Caveolin-1 overexpression is an early event in the progression of papillary carcinoma of the thyroid, $\mathrm{Br} \mathrm{J}$ Cancer 86 (2002), 912-916.

[22] S. Savin, D. Cvejic, T. Isic, I. Paunovic, S. Tatic and M. Havel$\mathrm{ka}$, Thyroid peroxidase and galectin-3 immunostaining in differentiated thyroid carcinoma with clinicopathologic correlation, Hum Pathol 39 (2008), 1656-1663.
[23] D. Kim, H. Kim and J.S. Koo, Expression of caveolin-1, caveolin-2 and caveolin-3 in thyroid cancer and stroma, Pathobiology 79 (2012), 1-10.

[24] T. Satoh, G. Yang, S. Egawa, J. Addai, A. Frolov, S. Kuwao, T.L. Timme, S. Baba and T.C. Thompson, Caveolin-1 expression is a predictor of recurrence-free survival in pT2N0 prostate carcinoma diagnosed in Japanese patients, Cancer 97 (2003), 1225-1233.

[25] S. Garcia, J.P. Dales, E. Charafe-Jauffret, S. CarpentierMeunier, L. Andrac-Meyer, J. Jacquemier, C. Andonian, M.N. Lavaut, C. Allasia, P. Bonnier and C. Charpin, Poor prognosis in breast carcinomas correlates with increased expression of targetable CD146 and c-Met and with proteomic basal-like phenotype, Hum Pathol 38 (2007), 830-841.

[26] H.J. Joo, D.K. Oh, Y.S. Kim, K.B. Lee and S.J. Kim, Increased expression of caveolin-1 and microvessel density correlates with metastasis and poor prognosis in clear cell renal cell carcinoma, BJU Int 93 (2004), 291-296. 


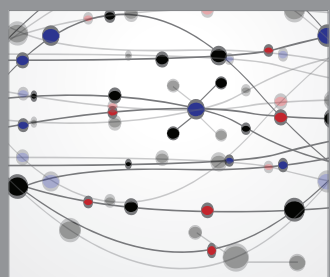

The Scientific World Journal
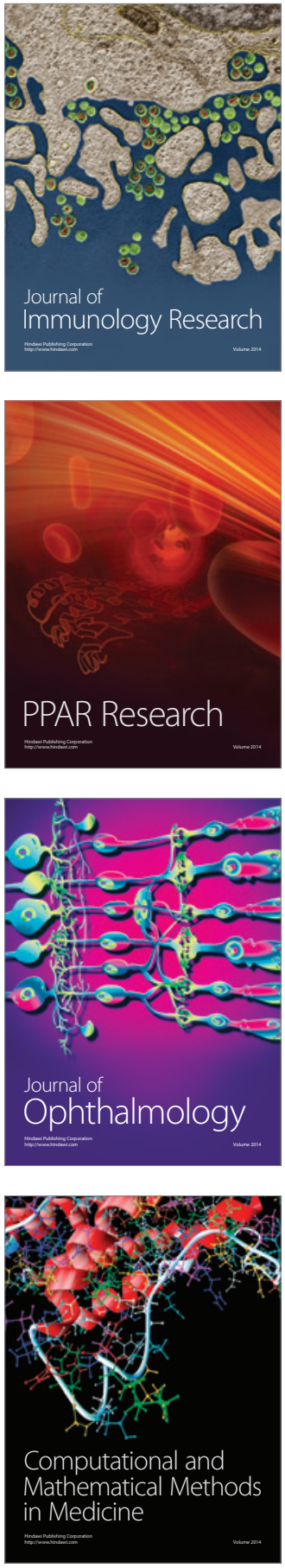

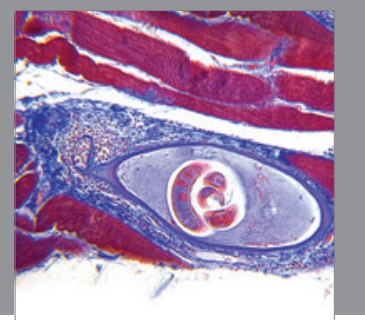

Gastroenterology

Research and Practice
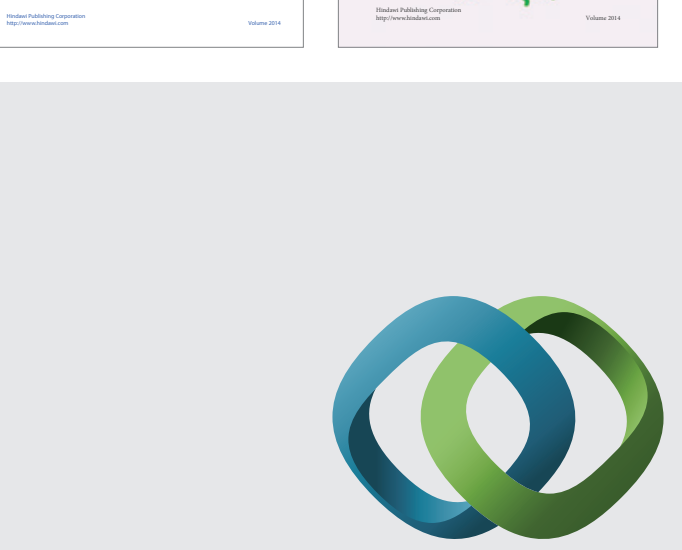

\section{Hindawi}

Submit your manuscripts at

http://www.hindawi.com
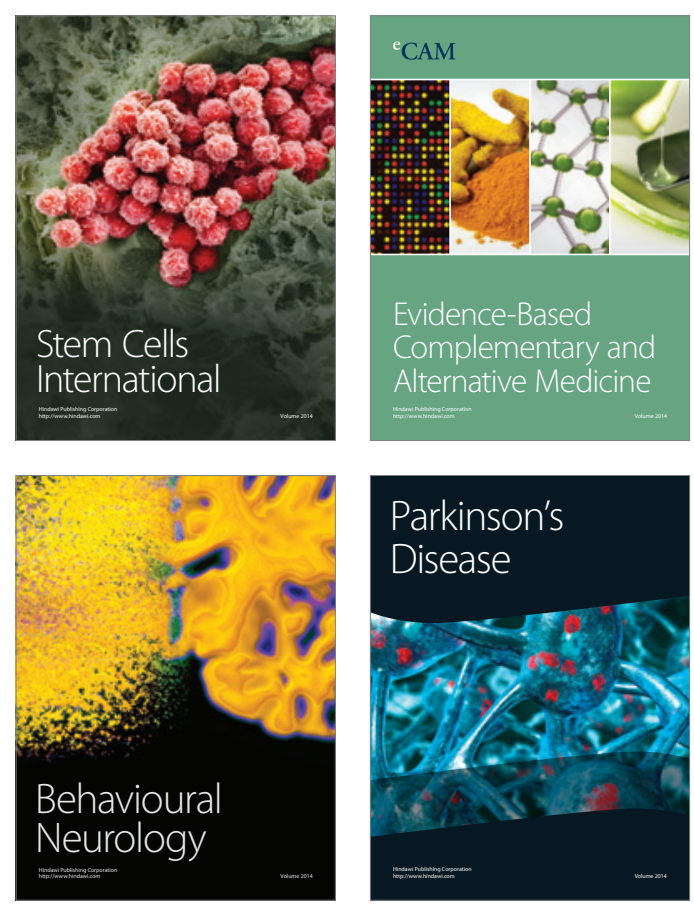

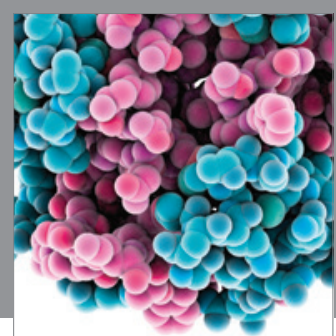

Journal of
Diabetes Research

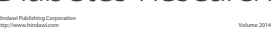

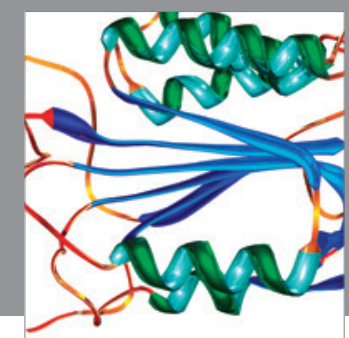

Disease Markers
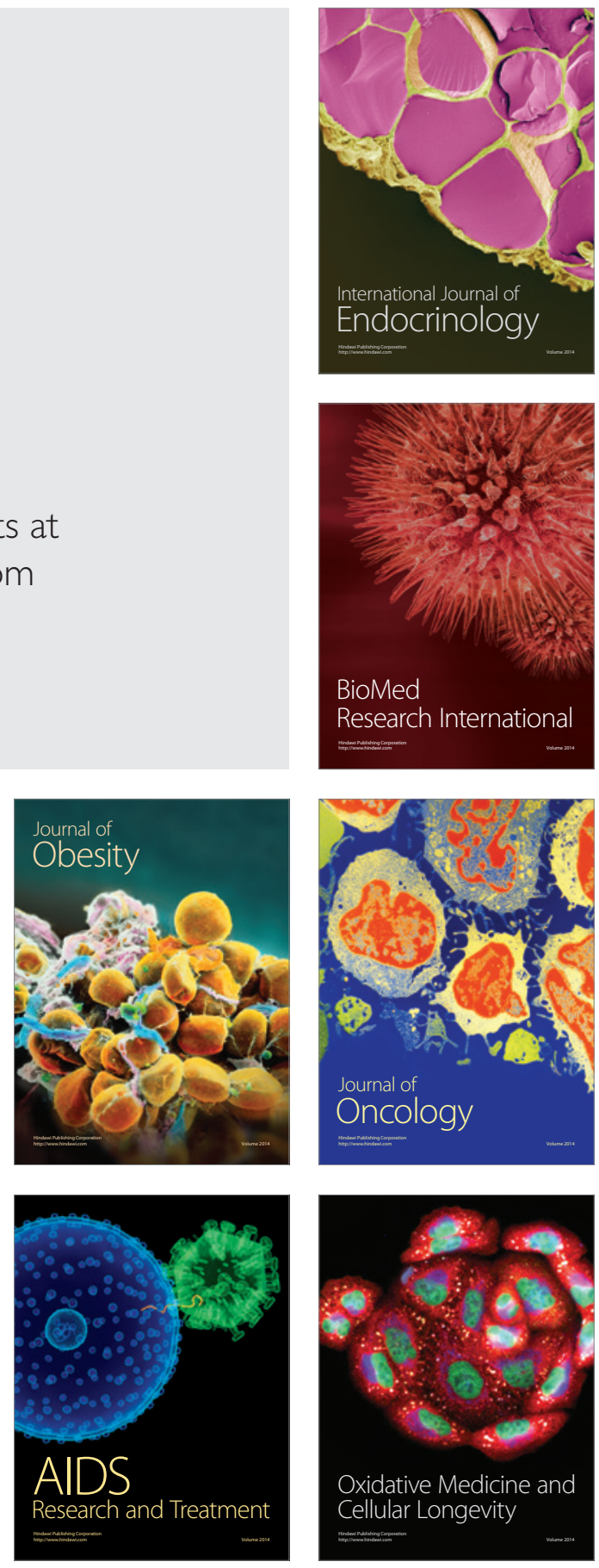